\title{
Damage Diagnostics in a Vertical Rod with Concentrated Masses on the Elastic Suspender
}

\author{
A. G. Khakimov \\ Institute of Mechanics, RAS Ufa Branch, Ufa, 450054, Russia
}

\begin{abstract}
Investigations have been performed to identify natural frequencies of longitudinal vibrations in a notched vertical rod with concentrated masses on the elastic suspender. It has been obtained that dependencies of the rod's circular longitudinal vibration frequencies on the notch coordinate have a periodic pattern. If the notch is located in the nodal point, the circular frequency of natural vibrations does not depend on the notch length. As shown, an increase in the mass of the lower load results in decreasing natural circular frequencies of the rod's longitudinal vibrations. The research into the problem shows that it is possible to determine the coordinate and dimensions of a transverse notch in the vertical rod using three frequencies of free longitudinal vibrations.
\end{abstract}

Keywords Sucker Rod, Vertical Sucker-Rod String, Concentrated Masses, Natural Frequencies Of Longitudinal Vibrations, Notch Parameter, Length Of Notch, Coordinate Of Notch

\section{Introduction}

A sucker-rod string is the major component that limits pump unit reliability and working capacity in directional holes. A significant amount of failures in sucker-rod strings is caused by rod breaks. A rod break is supposed to be preceded by the formation of a portion with a smaller cross-sectional area. In case of a finite-length bar, a change in the natural frequency of longitudinal vibrations can be used to detect its defects[1]. $\operatorname{In}[2]$ the solution is given for determining the variable cross-sectional area along the longitudinal coordinate from the known dependence of the bar's free-end displacement on the perturbing force frequency. $\operatorname{In}[3]$ and several other publications, a crack is simulated as a spring. A review of these studies is given in[4]. Different aspects of the problem are treated in[5, 6]. Paper[7] considers the solution to inverse problems on longitudinal travelling waves in finite-length bars.

$\operatorname{In}[8]$, the author uses three natural longitudinal vibration frequencies and thus determines locality and dimensions of a transverse notch in a vertical rod stretched out by gravity and the force applied to its lower end.

\section{Statement of the Problem}

Here we consider the stress-strain state of a straight rod fastened at its upper end to the elastic suspender with

* Corresponding author:

hakimov@anrb.ru (A.G. Khakimov)

Published online at http://journal.sapub.org/mechanics

Copyright (C) 2012 Scientific \& Academic Publishing. All Rights Reserved stiffness $c_{1}$ and stretched out by gravity and concentrated masses $M_{1}$ and $M_{2}$ (Figure 1). The rod is assumed to have a short portion (as compared to its overall length) with a smaller cross-section area. This notch does not cause any rod bending and simulates its damage, specifically of the open-crack type. Consideration is given only to the stress-strain state within the elastic limit for a thin rod. Since the crack results from a small nucleus, not necessarily in the most stressed section, the notch is assumed to be found at any place along the rod's length. The problem lies in determining the notch coordinate and dimensions relying on the approximation of the plane-section hypothesis.

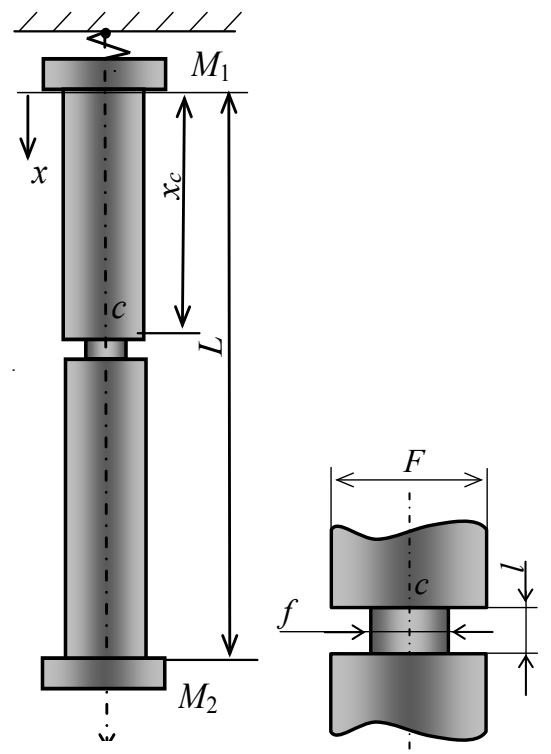

Figure 1. The design scheme 
Let us denote the length and area of the rod's cross section with $L, F$, the modulus of elasticity, density and coefficient of internal friction with $E, \rho, \mu$, the length and area of the notch's cross section with $l, f$, its coordinate with $x_{c}$ and the rod's displacement and force of tension with $u, T$. The following dependence is taken to be between stress $\sigma$ and deformation $\varepsilon$

$$
\sigma=E\left(\varepsilon+\mu \frac{\partial \varepsilon}{\partial t}\right), \quad \varepsilon=\frac{\partial u}{\partial x} .
$$

According to what has been said, we have

$$
E\left(\frac{\partial^{2} u}{\partial x^{2}}+\mu \frac{\partial^{3} u}{\partial x^{2} \partial t}\right)-\rho \frac{\partial^{2} u}{\partial t^{2}}=0, \quad T=E F\left(\frac{\partial u}{\partial x}+\mu \frac{\partial^{2} u}{\partial x \partial t}\right) .
$$

Let us measure the coordinate $x$ from the fastening point and write down the boundary conditions

$$
T=c_{1} u+M_{1} \frac{\partial^{2} u}{\partial t^{2}} \quad(x=0), T=-M_{2} \frac{\partial^{2} u}{\partial t^{2}} \quad(x=L) .
$$

There is a complex spatial stress-strain state in and near the notch of short length 1[9]. For the sake of simplicity, however, we assume uniaxial tension and compression. The experimental results[10] show that the mean value of the coefficient of longitudinal vibration attenuation in the notched rod under impact on its lower end is approximately 20 percent greater than the analogous coefficient for a non-notched rod. Let us consider the dynamic problem[1]

$$
\begin{aligned}
& \frac{\partial^{2} u}{\partial x^{2}}+\mu \frac{\partial^{3} u}{\partial x^{2} \partial t}-\frac{\rho}{E} \cdot \frac{\partial^{2} u}{\partial t^{2}}=0, \quad T=E F\left(\frac{\partial u}{\partial x}+\mu \frac{\partial^{2} u}{\partial x \partial t}\right) . \\
& T=c_{1} u+M_{1} \frac{\partial^{2} u}{\partial t^{2}} \quad(x=0), \quad T=-M_{2} \frac{\partial^{2} u}{\partial t^{2}} \quad(x=L) .
\end{aligned}
$$

Denoting the functions in the domains $0 \leq x \leq x_{c}, x_{c} \leq x \leq x_{c}+1$, $x_{c}+1 \leq x \leq L$ with indices $1,2,3$, respectively, let us write down the conditions for coupling solutions at $x=x_{c}$ and $x=$ $x_{c}+1$ (conditions for the equality of strains and displacements)[11]

$$
\begin{gathered}
\frac{\partial u_{1}}{\partial x}+\mu \frac{\partial^{2} u_{1}}{\partial x \partial t}=m\left(\frac{\partial u_{2}}{\partial x}+\mu \frac{\partial^{2} u_{2}}{\partial x \partial t}\right), \quad u_{1}=u_{2}, \quad\left(x=x_{c}\right), \\
m\left(\frac{\partial u_{2}}{\partial x}+\mu \frac{\partial^{2} u_{2}}{\partial x \partial t}\right)=\frac{\partial u_{3}}{\partial x}+\mu \frac{\partial^{2} u_{3}}{\partial x \partial t}, \quad u_{2}=u_{3}, \\
\left(x=x_{c}+l\right), \quad m=\frac{f}{F} .
\end{gathered}
$$

Conditions of form (3)-(4) are also given in[11] where the authors introduce a universal data processing procedure for the split Hopkinson bar[12]. Thus, the simplest notch model includes the coordinate $x_{c}$, length 1 and parameter $\mathrm{m}$. In the primal problem the coordinate $x_{c}$, length 1 and parameter $\mathrm{m}$ are known; in the inverse problem they should be found.

A particular solution to problem (1), at $\mu=0$, has the form $u=(A \cos \alpha x+B \sin \alpha x) \sin \omega t \quad\left(\alpha=\omega / a, a^{2}=E / \rho\right)$.

Six constants In this solution written down for the domains $0 \leq x \leq x_{c}, x_{c} \leq x \leq x_{c 1}, x_{c 1} \leq x \leq L\left(x_{c 1}=x_{c}+1\right)$ are determined from six boundary conditions (2)-(4). In order for $A_{i}, B_{i}$ $(i=1 \div 3)$ not to be simultaneously zero, the following determinant should equal zero

$$
\operatorname{det}\left(a_{i j}\right)=0 \text {, }
$$

where its non-zero elements are written down as

$$
\begin{aligned}
& a_{11}=c_{1}-M_{1} \omega^{2}, \quad a_{12}=-E F \alpha, \\
& a_{25}=E F \alpha \sin (\alpha L)+M_{1} \omega^{2} \cos (\alpha L), \quad a_{26}=-E F \alpha, \\
& a_{31}=\sin \left(\alpha x_{c}\right), a_{32}=-\cos \left(\alpha x_{c}\right), a_{33}=-m \sin \left(\alpha x_{c}\right), a_{34}=m \cos \left(\alpha x_{c}\right), \\
& a_{41}=\cos \left(\alpha x_{c}\right), a_{42}=\sin \left(\alpha x_{c}\right), a_{43}=-\cos \left(\alpha x_{c}\right), a_{44}=-\sin \left(\alpha x_{c}\right), \\
& a_{53}=m \sin \left(\alpha x_{c l}\right), a_{54}=-m \cos \left(\alpha x_{c l}\right), a_{55}=-\sin \left(\alpha x_{c l}\right), a_{56}=\cos \left(\alpha x_{c l}\right), \\
& a_{63}=\cos \left(\alpha x_{c l}\right), a_{64}=\sin \left(\alpha x_{c l}\right), a_{65}=-\cos \left(\alpha x_{c l}\right), a_{66}=-\sin \left(\alpha x_{c l}\right),
\end{aligned}
$$

Condition (5) gives a frequency equation not presented here because of its awkwardness.

When the coefficient is $c_{1} \rightarrow \infty$, the frequency equation simplifies.

For the non-notched rod without concentrated masses it follows from the frequency equation that $\cos \alpha L=0$ and natural frequencies equal[1] $\alpha L=(2 k-1) \pi / 2(k=1,2, \ldots)$ or $\omega_{k}=(2 k-1) \pi a / 2 L$.

The determination of $m, l$ and $x_{c}$ calls for an analysis of natural longitudinal vibration frequencies in the notched rod to be done.

\section{Primal problem}
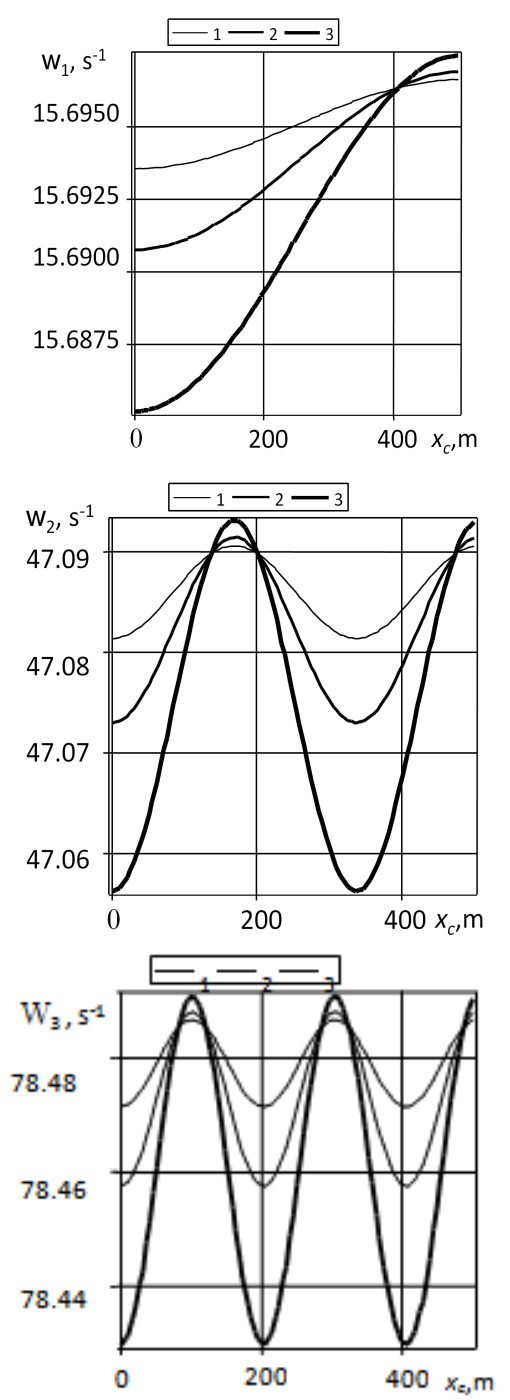

Figure 2. Dependences of the rod's circular longitudinal vibration frequencies $\omega_{1}, \omega_{2}, \omega_{3}$ on the notch coordinate $x_{c}$ for the parameter $m=0.1$ and different lengths $l$ (curve $1-0.01,2-0.02,3-0.04 \mathrm{~m}$ ) 

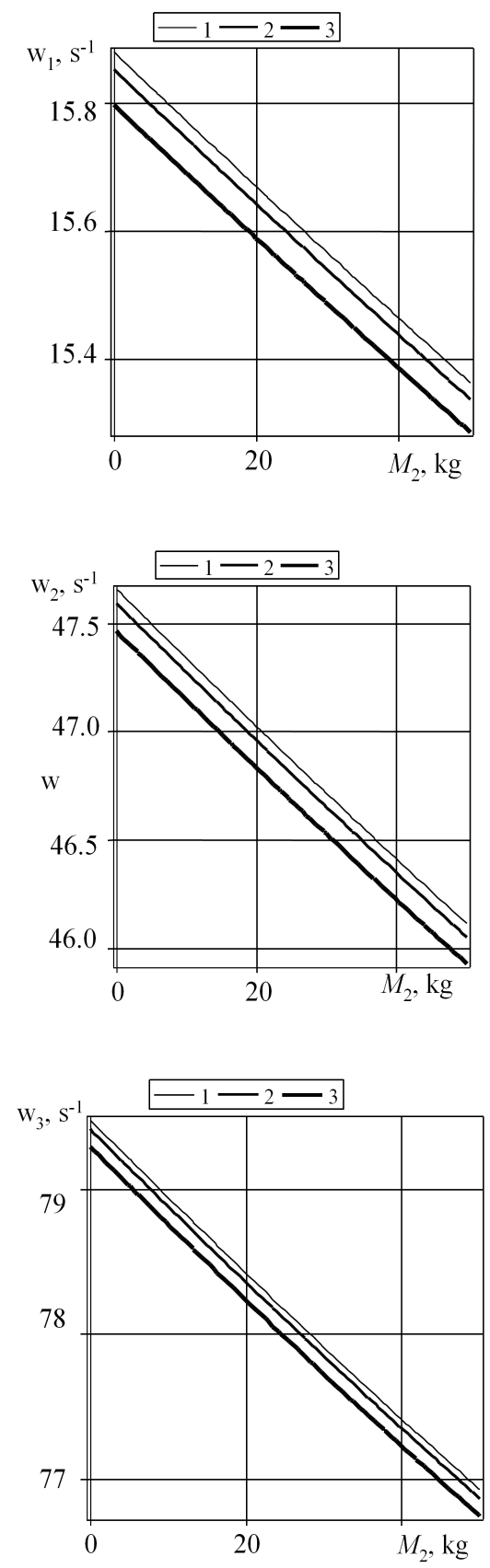

Figure 3. Dependences of the rod's circular longitudinal vibration frequencies $\omega_{1}, \omega_{2}, \omega_{3}$ on the mass $M_{2}$ for the notch coordinate $x_{c}=50 \mathrm{~m}$, parameter $m=0.1$ and different lengths $l$ (curve $1-0.1,2-0.2,3-0.4 \mathrm{~m}$ )

The solution to the frequency equation has been made numerically for the following parameters of the system: $E=$ $2 \cdot 10^{11} \mathrm{~Pa}, \rho=7800 \mathrm{~kg} / \mathrm{m}^{3}, L=500 \mathrm{~m}, F=0.00038 \mathrm{~m}^{2}$ (rod's diameter is $22 \mathrm{~mm}), c_{1} \rightarrow \infty, M_{1}=20 \mathrm{~kg}, M_{2}=20 \mathrm{~kg}$. The speed of sound is $a=5063.6 \mathrm{~m} / \mathrm{s}$. In this case the first, second and third natural frequencies of the non-notched rod are $\omega_{1}=$ 15. $6963 \mathrm{~s}^{-1}, \omega_{2}=47.0897 \mathrm{~s}^{-1}, \omega_{3}=78.4853 \mathrm{~s}^{-1}$. For the notched rod at $x_{c}=100 \mathrm{~m}, m=0.1,1=4 \mathrm{~m}$ the solution to the primal problem gives that circular longitudinal vibration frequencies are $\omega_{1}=14.7787 \mathrm{~s}^{-1}, \omega_{2}=46.2813 \mathrm{~s}^{-1}, \omega_{3}=$ $79.0466 \mathrm{~s}^{-1}$. Figure 2 shows the dependences of the rod's circular longitudinal vibration frequencies $\omega_{1}, \omega_{2}, \omega_{3}$ on the notch coordinate $x_{c}$ for the parameter $m=0.1$ and different lengths 1 (curve $1-0.01,2-0.02,3-0.04 \mathrm{~m}$ ). These dependences have periodic patterns. If the notch is located in the nodal point, the circular frequency of natural vibrations does not depend on the notch length.

Figure 3 shows the dependences of the rod's circular longitudinal vibration frequencies $\omega_{1}, \omega_{2}, \omega_{3}$ on the mass $M_{2}$ for the notch coordinate $x c=50 \mathrm{~m}$, parameter $m=0.1$ and different lengths 1 (curve $1-0.1,2-0.2,3-0.4 \mathrm{~m}$ ). As can be seen, at a given notch coordinate an increase in the mass $M_{2}$ results in decreasing natural circular frequencies of the rod's longitudinal vibrations.

\section{Inverse Problem}
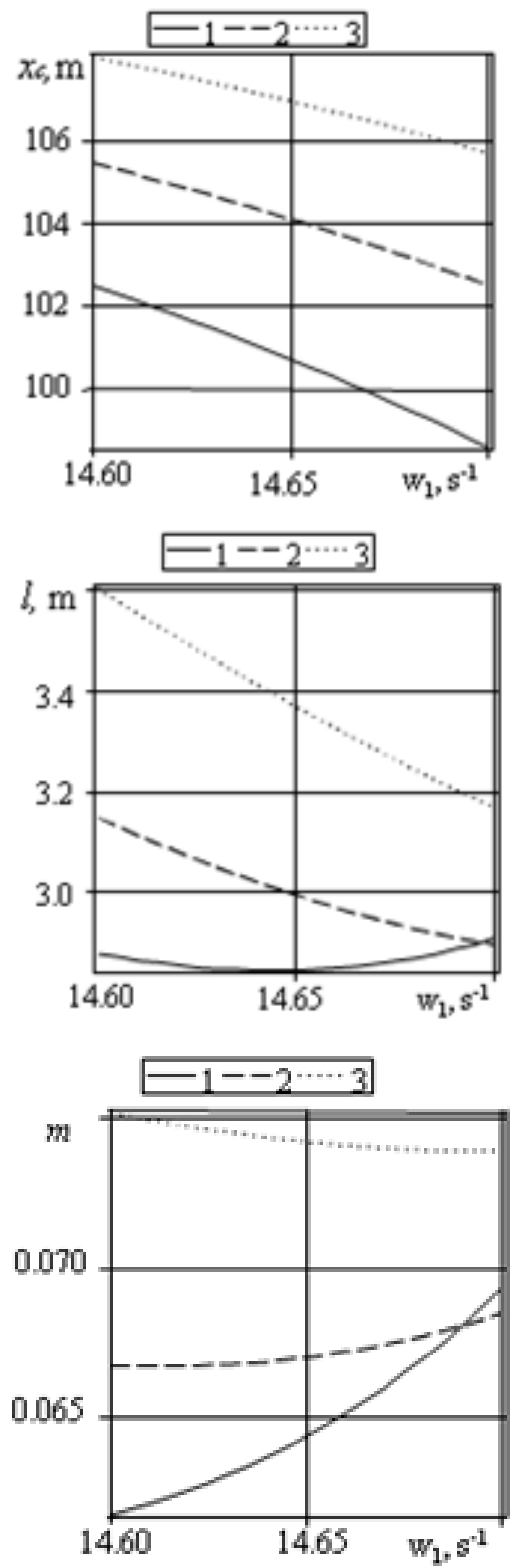

Figure 4. Dependences of the notch coordinate $x_{c}$, length $l$ and parameter $m$ on the rod's circular longitudinal vibration coordinate $\omega_{1}$, for $\omega_{2}=46.1$ $\mathrm{rad} / \mathrm{s}$ (curve 1), $\omega_{2}=46.2 \mathrm{rad} / \mathrm{s}$ (curve 2), $\omega_{2}=46.3 \mathrm{rad} / \mathrm{s}$ (curve 3$), \omega_{3}=78.9$ $\mathrm{rad} / \mathrm{s}$ 
Writing the frequency equation for three frequencies of free longitudinal vibrations, we can use the resulting set of equations to determine the notch coordinate $x_{c}$, length $l$ and parameter $m$. For example, for the rod's circular longitudinal vibration frequencies $\omega_{1}=14.6 \mathrm{~s}^{-1}, \omega_{2}=46.1 \mathrm{~s}^{-1}, \omega_{3}=78.9 \mathrm{~s}^{-1}$ the solution of the inverse problem gives that the rod has a notch at $x_{c}=102.51 \mathrm{~m}, m=0.062, l=2.878 \mathrm{~m}$.

Figure 4 shows the dependences of the notch coordinate $x_{c}$, length $l$ and parameter $m$ on the rod's circular longitudinal vibration frequencies $\omega_{1}$, for $\omega_{2}=46.1 \mathrm{rad} / \mathrm{s}$ (curve 1), $\omega_{2}=$ $46.2 \mathrm{rad} / \mathrm{s}$ (curve 2), $\omega_{2}=46.3 \mathrm{rad} / \mathrm{s}$ (curve 3 ), $\omega_{3}=78.9 \mathrm{rad} / \mathrm{s}$.

\section{Conclusions}

Dependencies of the rod's circular longitudinal vibration frequencies on the notch coordinate have a periodic pattern. If the notch is located in the nodal point, the circular frequency of natural vibrations does not depend on the notch length.

An increase in the mass of the lower load results, at a given notch coordinate, in decreasing natural circular frequencies of the rod's longitudinal vibrations.

The coordinate and dimensions of a transverse notch in the vertical rod hanged on the elastic suspender and stretched out by gravity and concentrated loads are determined using three frequencies of free longitudinal vibrations.

The obtained results can be recommended for diagnosing damages in sucker-rod strings.

\section{ACKNOWLEDGEMENTS}

The investigation was financially supported by the Russian Foundation for Basic Research (Projects 11-01-97003-r_povolzhie_a, 11-01-00293_a).

\section{REFERENCES}

[1] M.A. Ilgamov, "Damage Detection in Vertical Rod", Inst. Mechanics, RAS Ufa Branch, Transactions 5, pp. 201-211, 2007.

[2] A.O. Vatulyan, Inverse Problems in Deformed Solid Mechanics, Fizmatlit Publ., Russia, 2007.

[3] G. Biscontin, A. Morassi, P. Wendel, "Asymptotic Separation of Spectrum in Notched Rods", Journal of Vibration and Control, vol. 4, no. 3, pp. 237-251.

[4] G.M.L Gladwell, Inverse Problems in Vibration, Kluwer Academic Publishers, Germany-USA-UK, 2004.

[5] T. Pritz, "Apparent Complex Young's Modulus of a Longitudinally Vibrating Viscoelastic Rod", Journal of Sound and Vibration, vol. 77, no. 1, pp. 93-100, 1981.

[6] D. Benatar, D. Rittel, A. L. Yarin, "Theoretical and Experimental Analysis of Longitudinal Wave Propagation in Cylindrical Viscoelastic Rods", Journal Mechanics and Physics of Solids, vol. 51, no. 8, pp. 1413-1431, 2003.

[7] A.O. Vatulyan, N.O. Soluyanov, "On Determining the Cavity's Location and Size in Elastic Bar", Journal of Defectoscopy, no. 9, pp. 44-56, 2005.

[8] A.G. Khakimov, "Damage Diagnostics in a Vertical Bar on the Elastic Suspender, Electronic Journal on Oil and Gas Engineering, 2010. On-line available: http://www.ogbus.ru/authors/Khakimov/Khakimov_1.pdf.

[9] V.Z. Parton, E.M. Morozov, The Mechanics of Elastic-Plastic Destruction, Nauka Publ., Russia, 1974.

[10] A.O. Ryazyantsev, "Vibro-Acoustic Detection of Deep-Well Sucker Rods during Operation", M. Eng. Thesis, Ufa State Petroleum Technical University, Russia, 1999.

[11] S.L. Lopatnikov, B.A. Gama, C. Krauthauser, J.W. Gillespie Jr., "On the Applicability of the Classical Analysis of Experiments with Split Hopkinson Bar", Technical Physics Letters, vol. 30, no. 3, pp. 39-46, 2004.

[12] H. Kolsky, "An Investigation of Mechanical Properties of Materials at Very High Rates of Loading", in Proceedings of the Physics Society of London, vol. 62, no. 359, pp. 676-700, 1949. 